\title{
Delirium Incidence, Duration and Severity in Critically III Patients with COVID-19
}

Jessica Hammes ${ }^{1}$, Sikandar Khan ${ }^{2,4}$, Heidi Lindroth ${ }^{2,3,4}$; Babar Khan $2,4,5,6$

${ }^{1}$ Indiana University School of Medicine Indianapolis, IN

${ }^{2}$ Division of Pulmonary, Critical Care, Sleep and Occupational Medicine, Department of Medicine, Indiana University School of Medicine, Indianapolis, IN

${ }^{3}$ Indiana University School of Nursing, Indianapolis, IN

${ }^{4}$ IU Center of Aging Research, Regenstrief Institute, Indianapolis, IN

${ }^{5}$ Indiana University Center of Health Innovation and Implementation Science, Indianapolis, IN

${ }^{6}$ Sandra Eskenazi Center for Brain Care Innovation, Eskenazi Hospital, Indianapolis, IN

\section{Background:}

COVID-19 is associated with severe respiratory failure and high mortality in critically ill patients. ${ }^{2,4,5}$ Neurologic manifestations of the disease, including delirium and coma, may also be associated with poor clinical outcomes. Delirium is associated with prolonged mechanical ventilation and mortality. ${ }^{3}$ this study sought to describe the rates, duration, and severity of delirium in patients admitted to the ICU with COVID-19.

\section{Methods:}

A retrospective, observational study was conducted from March $1^{\text {st }}$ to April $27^{\text {th }}, 2020$, at Indiana University Health Methodist and Eskenazi Health Hospitals. The delirium measurements were extracted in the first 14 days of the ICU stay, using the Richmond Agitation and Sedation Scale (RASS) and the CAM-ICU and CAM-ICU7, for those with a positive COVID-19 diagnosis. The primary outcomes were delirium rates and duration; the secondary outcome was delirium severity. Descriptive statistics and median group comparisons were done using SAS v9.4.

\section{Results:}

Of 144 patients in the study, $73.6 \%$ experienced delirium and $76.4 \%$ experienced delirium or coma. The median delirium or coma duration was 7 days (IQR: 3-10), and the median delirium duration was 5 days (IQR: 2-7). The median CAM-ICU-7 score was 6 (IQR: 2-7) signifying severe delirium. Mechanical ventilation was associated increased risk of developing delirium (OR: 22.65, 95\% CL: 5.24-97.82). Mortality was also more likely in patients experiencing delirium: $26.4 \%$ compared to $15.8 \%$ in patients without delirium.

\section{Conclusion:}

Of the 144 patients included, $73.6 \%$ experienced delirium lasting on average 5 days: the median delirium score being severe. Mechanical ventilation was also associated with greater odds of developing delirium. Because Covid-19 is associated with high rates of delirium, leading to increased rates of functionality disability, more resources and attention are needed to prevent and manage delirium in patients. ${ }^{1}$ 


\section{References}

1. Brummel NE, Jackson JC, Pandharipande PP, et al. Delirium in the ICU and subsequent long-term disability among survivors of mechanical ventilation. Critical Care Medicine. 2014;42(2):369-377.

2. Grasselli G, Pesenti A, Cecconi M. Critical Care Utilization for the COVID-19 Outbreak in Lombardy, Italy: Early Experience and Forecast During an Emergency Response. JAMA. 2020.

3. Hayhurst CJ, Pandharipande PP, Hughes CG. Intensive Care Unit Delirium: A Review of Diagnosis, Prevention, and Treatment. Anesthesiology. 2016;125(6):1229-1241.

4. Li YC, Bai WZ, Hashikawa T. The neuroinvasive potential of SARS-CoV2 may play a role in the respiratory failure of COVID-19 patients. J Med Virol. 2020.

5. Wu Y, Xu X, Chen Z, et al. Nervous system involvement after infection with COVID-19 and other coronaviruses. Brain, behavior, and immunity. 2020:S0889-1591(0820)3035730353. 